\title{
Machine Learning-Based Optimized Hierarchical Routing Protocols for WSN Lifetime: A Review
}

\author{
P.Suman Prakash ${ }^{1}$, D. Kavitha ${ }^{2}$, P. Chenna Reddy ${ }^{3}$ \\ ${ }^{1}$ Research Scholar,Jawaharlal Nehru Technological University Anantapur, Andhra Pradesh, India \\ ${ }^{2}$ Department of Computer Science and Engineering, G.Pulla Reddy Engineering College, Kurnool, India \\ ${ }^{3}$ Department of Computer Science and Engineering, Jawaharlal Nehru Technological University Anantapur, Andhra Pradesh,
} India

\begin{abstract}
In Wireless Sensor Networks, network lifetime optimization has challenging and significant issue. Subsequently, most of the existing works delineate several factors to improve the network lifetime: by decreasing the amount of the consumption of energy, reducing latency, load balancing, clustering, efficient data aggregating and by minimizing the data transmission delays. This paper provides a review of recent techniques and presents a Machine Learning-based Optimized Hierarchical Routing Protocols for WSN Lifetime. Research has been done, and reviews have been studied to explore the energy management schemes using optimized routing approach and Machine Learning Adaptability for WSN's. Further, recommend future directions related to the Optimized Clustering Approaches to enhance wsn lifetime.
\end{abstract}

Keywords: Wireless Sensor Networks, Network Lifetime, Machine Learning, Clustering Techniques, neural network, Routing Algorithms

\section{Introduction}

In recent years, many have involved in quality research in Wireless Sensor Networks within the community due to enrich the QoS and enhancing the network lifetime. A WSN is organized by spatially distributed independent devices such as a massive volume of sensor nodes communicated wireless, and without human intervention to accumulate and transmit the data between sensor nodes to the Base Station. These networks are limited in range with decreased battery life in terms of processing power, memory capacity, power source, and so on. The communication process, in WSN, is through neighboring nodes to reach the base station. Nodes are usually predictable to function based on battery lifetime. Renovation or recharging the batteries of sensor nodes in the region is expensive and effortful. Direct extensive network and may reduce the network lifetime considerably.

Energy Efficiency or increasing the life time of a WSN is a complicated problem and can be handled by a combination of much advancement in different layers of WSN protocol stack. Energy efficient MAC, energy efficient routing, effective congestion detection algorithms all play a part. Even the placement of nodes in the WSN is important some papers formulated mathematical formulation for the optimal node distribution strategy. Some researches worked on the use of relay nodes whose action is only to relay packets and not sensing and even the placement of those relay nodes depending on the distance from the Master Station are important. Efficient Cluster head selection, the size of cluster and frequency in updating the cluster member parameters all play a part in an efficient energy WSN design. Some even use data aggregation and others use on/off techniques for energy savings.

The methods of [1] may offer a viable solution for the consumption of is involved in getting a solution to this issue. Likewise, an alternative method, i.e., "hierarchical or clusterbased routing," would be very beneficial techniques for largescale WSNs to overcome the shortcomings of scalability, efficient communication, and fault tolerance. The complete network system is scattered into sub-networks in hierarchical architectures, named clusters. Respectively, a unique node named "CH".

The purpose of this paper is to conduct a systematic literature survey on "Optimized Hierarchical Routing Protocols" for "WSN Lifetime using Machine Learning-based approaches." This is still an area of active research investigating the scheme of efficient routing protocol at the network layer that can address the assumption of energysaving methodologies at the data link layer to attain energyawareness in WSNs. With this aim in mind, this paper enlightens the present optimized energy-aware communication in WSNs using machine learning techniques. Consequently, machine learning-based clustering is achieved credible by exploiting methods founded on recent research on Machine Learning (ML). These modern clustering models are known as improved clustering algorithms. They lead the expected clustering solutions in terms of "scalability, reliability, fault tolerance, amount of data delivered, energy consumption, better coverage of the experimental field, and the intensification of the network lifetime."

The characteristics of WSN [4]:

Computing abilities: The sensor's program and storage space are minimal due to the size, cost, and battery consumption. 
Battery energy: Due to battery exhaustion, the sensor nodes are often invalid and out of control. Consequently, the protocols and algorithms should be considered for the conservation to be considered in advance.

Communication capabilities: WSN Communication devices' capabilities are limited due to their limited communication capabilities and range. As a rule, the distance of the communication range is less than one meter, while the sensor is affected by influences from the natural environment.

Dynamic Power Management: DPM is a way of controlling and saving energy depletion at the sensor node. Sensor nodes are equipped with batteries that serve as an energy source, and energy consumption depends on whether the sensor node is deployed for acquisition, data processing, or communication. The sensor network cannot be employed for amore extended period, making it challenging to charge or replace the batteries.

Multi-hop communications: In WSN, communication happens among the nodes within radio frequency, making the intercommunication through multi-hop routing transmission.

Application relevance: It focuses on traffic patterns from WSN data aggregation and many more. The sensor network differs from traditional networks in that it relies on applications and its ultimate working environment to receive data. Different sensor network applications process different physical signals and routing protocols that are not applied to one another.

Reliability: Packet size, node density, Protocol design, hop-by-hop communication, end-to-end communication, buffer length, packet loss ratio, packet delivery ratio, throughput, and loss recovery are the key factors that affect reliability in WSN [5].

Reliability semantics: Packet reliability anticipates successful transmission of entire packet transmission. Apart from event reliability, end-to-end reliability, up-stream reliability, and down-stream reliability are also key factors in defining the reliability semantics.

General Issues in WSN:Node Deployment: The first and foremost challenge is node deployment, and there are several obstacles to this process due to environmental conditions and geographical location; it is expensive [5].

Energy consumption without accuracy: when there is an establishment of communication in the network from node to $\mathrm{BS}$ based on the communication range, a sum of units of nodes is deployed in the network causes the consumption of which may affect the total network lifetime.

Coverage: The sensor covers only partial filed of the physical area in the network due to limited visual accuracy in the environment.

Node Dynamics: There is an assumption that the nodes are stable in network structures. However, sometimes, it is imperative to reinforce the flexibility of base stations or CHS. The dynamics of a node is a very confronting task because node membership comes to be dynamic, forcing the cluster over time.

Overlay Cluster: As mentioned previously, the $\mathrm{CH}$ can be predefined by the network admin or selected by the sensors in the network. If one chooses later, a member of one cluster is likely to become a member under another $\mathrm{CH}$. It overlaps groups to consider design issues. Therefore, it is essential to establish the mechanisms necessary to identify the presence of overlapping groups and to coordinate between groups to prevent injustice, hunger, or stagnation during resource competition.

Load Balancing: It is a significant problem in sensor networks, where $\mathrm{CHs}$ are raised after the available sensors. Member sensor nodes need to be delivered evenly among the various available $\mathrm{CHs}$, and if they fail, the head overloads the specific $\mathrm{CH}$ for failure. So in such cases, it is necessary to create groups of equal size for fair balance.

Other parameters that are influential for cluster design are Dynamic Cluster Control, Inter-cluster Coordination, Data Aggregation, Fault Tolerance, Scalability, Number of Clusters, Cluster Formation Time; Single hop Vs. Multi hops Network, Heterogeneity, Cluster Formation, SelfConfiguration, and Reconfiguration [7].

Design Issues:Energy Consumption: Energy consumption is a severe problem in the WSN, for every effective communication to deliver and to take the message among the nodes to base station requires energy. Every sensor node initially has equal energy levels; depends on the participation of nodes in the communication, their energy gets depleted. Eventually, energy consumption varies on the complex operation of sensor nodes, namely "sensing, data processing, communication." Therefore, effective routing protocols must be deployed in every way to prevent energy consumption.

Localization: It is an essential parameter to perform the operation and resource management in WSN. Due to the improvised nature of the sensor nodes in WSN, storing node location information remains a challenging issue in the network. This difficulty can be resolved by using ideal nodes, GPS, and proximity-based localization. Other design issues affecting the lifetime of WSN include coverage, clocks, counts, product costs, hardware design, and service quality.

Coverage: In coverage of WSNs, every sensor node inspects the environment. It only covers a controlled substantial area of the environment. Consequently, area coverage is also a noteworthy design parameter in WSNs.

The atmosphere of the sensor is imprecise and covers only a controlled physical area of the background. By covering a WSN, the sensor node receives a static view and covers a limited physical area of the environment. Other design problems affecting the lifetime of WSNs include the presence of other sensors in the same area, such as a sensor in another location, and the absence of localization protocols. 
Connectivity: It depends on a random scattering of nodes, so area coverage is also an important design parameter for WSNs. Therefore, sensor nodes are regarded to be strongly networked. Moreover, network size reduces sensor node failure. However, the network does not avert the network topology since this topology is variable and can bring about network failure in the event of sensor failure.

Fault-Tolerance: A high degree of redundancy is required. One sensor node's failure should not influence the overall performance of the sensor network [5]. However, in the event of a sensor failure, sensor nodes can be blocked, which can cause the network to fail even if connected.

Scalability: The routing protocol of the sensor network is sufficient to react to outcomes in the condition. Besides, Routing systems should be able to work with a large number of sensor nodes and can be with sensor ranges in the order of hundreds of thousands or more. Most sensors sleep until an event occurs, but the data from the remaining sensors provide a coarse quality.

This paper presents the following contributions:

- In this study, validated methods were deployed to assess the existing intelligent clustering approaches.

- We explored the influence of Machine Learning-Based Classification algorithms in WSN;

- Accurate evaluation and comparison of selected algorithms, key parameters.

The remainder of this paper consists of as follows: Section 2 Describes Routing on Wireless Sensor Networks. Section 3presents a Comparison of the Optimized Clustering Approaches. Section 4 presents Mathematical approaches for WSN lifetime enhancement. Section 5 presents Machine Learning Adaptability for WSN's; section 6, there remains several issues that should be explored in future research and Section 7. The conclusion of this paper is summarized here.

\section{Routing On Wireless Sensor Networks}

Routing is an option of extending the network lifetime by regulating the node energy consumption under certain conditions. In WSN, several protocols are fundamentally concerned with the maintenance of network lifetime. It depends on the design of the network and routing. Herein, two approaches were employed. In the first locationbased routing. The second involves group-based routing. This classification is supported by network architecture.

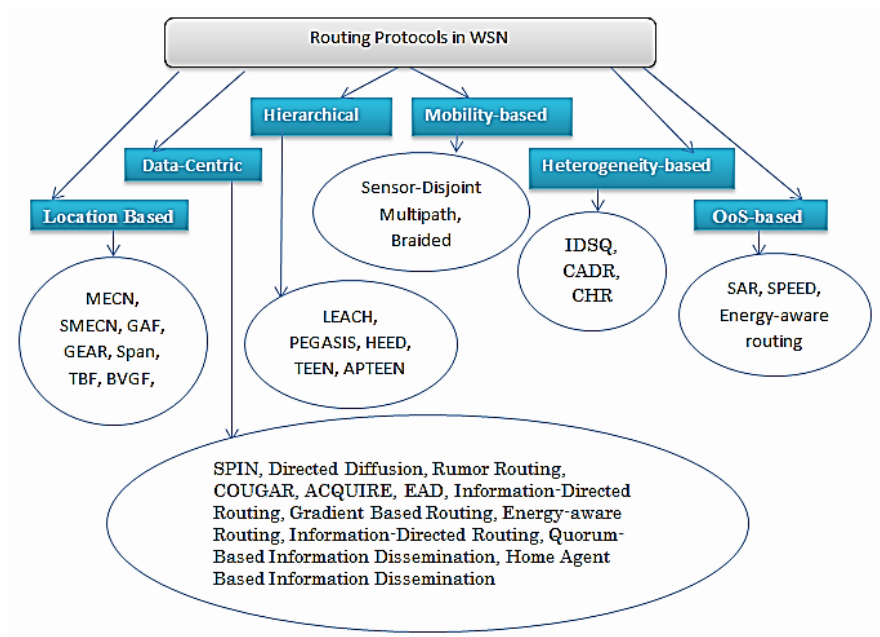

Figure 1. Routing Protocols in WSN

\subsection{Routing Approach}

It was found that such an approach can be classified as two methodologies i.e.

Classical-Based Routing: In traditional routing nodes in the primary cluster are randomly selected using a timer function that creates an irregular flow of traffic between the different primary nodes it leads to several challenging issues, such as "scalability; Load balancing, connectivity, coverage, and rigor."

Optimized-Based Routing: It shows a pivotal role in improving the life of the WSN network. Lack of tolerance, lack of energy efficiency, connectivity, rigidity, and scalability are the main barriers in this system.

\subsection{Clustering Characteristics}

The characteristics of the clustering process likely to influence hierarchical-based routing efficiency. Cluster characteristics and sensor capabilities are two significant problems in clustering properties $[8,9]$.

\subsection{Clustering objectives:}

Enhancing the lifetime of the network: As the presentation of $\mathrm{SN}$ is robustly depends on the network lifetime, in this regards to investigate the way to use node energy supply efficiently as a concern routing algorithms outcome in reduce energy depletion and enhanced bandwidth utilization.

Fault-tolerance: To address the issue of a node failure, various techniques are designed. To cope with this issue, either the concept of proxy $\mathrm{CH}$ is deployed (when either node fails or has less transmission energy), or $\mathrm{CH}$ rotation can be the solution. Fault tolerance is one of the essential objectives while designing the clustering algorithm.

Fill handling: Fill handling is another outline objective while designing a clustering protocol. It is unquestionably required not to overweight the $\mathrm{CHs}$ as it leads to quick depletion of energy. So in each cluster, even 
distribution of node is essential, as $\mathrm{CHs}$ performs the task aggregation of data or other signal handling task.

\subsection{Cluster Head Selection Criteria}

To select a $\mathrm{CH}$, the following parameters are taken into account: Distance: Selection of $\mathrm{CH}$ largely affects WSNs lifetime, distance is the one of the key parameter in selection of $\mathrm{CH}$ in the sensor network, i.e. calculate the distance among the source nodes to the base station.

Residual Energy: This energy is very much necessary to select the $\mathrm{CH}$. This energy seems to be the remaining energy of the sensor after communication. This RE decreases the node energy during communication between the $\mathrm{CH}$ and BS. Consequently, for every new round of communication, the $\mathrm{CH}$ is elected depends on the RE.

Network Average Energy: This energy is deployed as reference energy for every node. This is aggregate energy in sensor nodes. Nodes with high energy will only participate in $\mathrm{CH}$ selection [49]

\subsection{Cluster Properties}

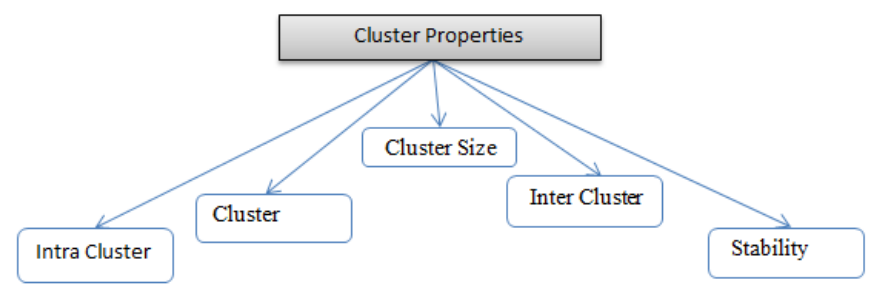

Figure 2. Cluster Properties

Cluster Size: cluster size impact the WSN lifetime by considering the number of nodes and its transmission range. There is a possibility of node energy depletion if the number of nodes in the cluster is increased. When many nodes in the cluster are more, it may lead to node energy depletion upon more number of iterations.

Cluster Density: It represents the total cluster member, which affects the energy consumption of $\mathrm{CH}$. This cluster density may vary on the type of the cluster i.e., static or dynamic.

Intra Cluster Routing: Communication between the cluster members to the CHs. There is single hope communication between the $\mathrm{CM}$ to $\mathrm{CH}$ depends on the network structure. There is a possibility of data redundancy from the cluster members.

Inter-Cluster Routing: Typically communication amid the $\mathrm{CH}$ to the base station is done determined the single hop or Multi-hop routing with the use of intermediate nodes.

Stability: The routing process stability is resolved when the cluster density is constant.

\subsection{The Specific Steps of Clustering Routing Protocol}

1) Determination of the number of cluster-head: To determine the clustering number $\mathrm{K}$ based on the mathematical model and the formula, then get clustering probability $\mathrm{P}=\mathrm{K} /$ N.

2) Preliminary clustering: Firstly, the network should be initialized and preliminary clustered with LEACH, determining the head's temporary clusters and cluster. Simultaneously, the temporary $\mathrm{CH}$ collects the state information of each member in the cluster, including location information (spatial coordinates) and energy information.

3) Optimize the temporary cluster with the QPSO algorithm: The population size of quantum particles are initialized with cluster number $\mathrm{K}$, and the spatial coordinates of the sensor nodes correspond to the position of quantum particles.

4) Clustering after optimization: The temporary $\mathrm{CH}$ broadcasts the information of formal cluster-head to the nodes in the cluster, and then the clustering in this round and the determination of cluster-head are finished.

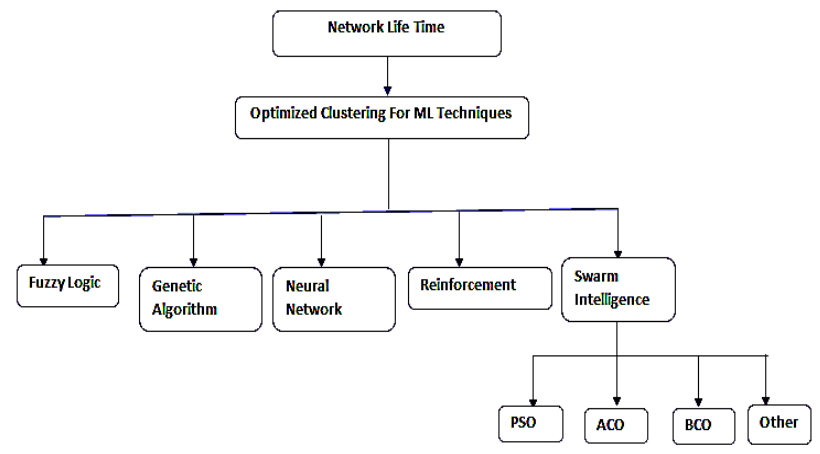

\section{Figure 4: Classification of ML/CI paradigms deployed in} clustering in WSNs

\subsubsection{Fuzzy Logic}

Fuzzy logic systems (FLS) mainly consisting four parts such as fuzzification, inference engine, Rule-based, and defuzzification.

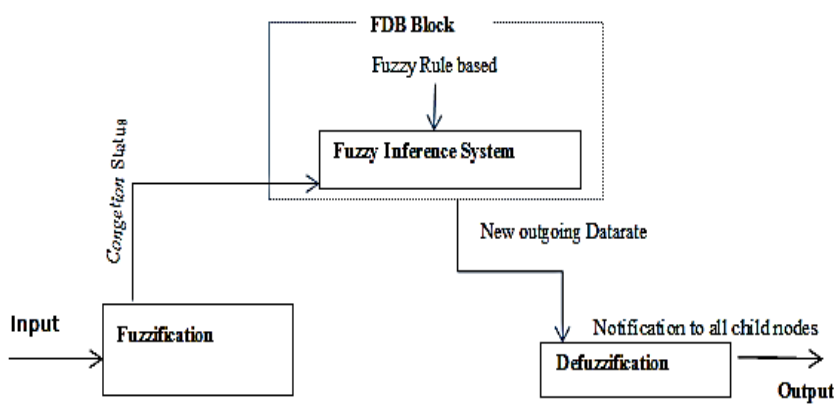

Figure 5.Consisting of fuzzifier, fuzzy decision block (FDB), and fuzzified block

The first step is to enter the data and use the present functions to determine the degree of each relevant fuzzy set. 
Fuzzy inference is the process of generating drawing from input to a given output using fuzzy logic. The representing then provides guidelines for decision-making or model identification. Degradation is the process of converting the fuzzy output of a fuzzy inference system into a pure output.

Most of the researchers ensure that how Fuzzy Logic can be utilized to choice the proper and efficient $\mathrm{CH}$ so that an efficient lifetime can be consummate. There are many Clustering algorithms, but the Fuzzy logic algorithm is mostly deployed.

\section{FCH:}

Selection of $\mathrm{CHs}$ using fuzzy logic $(\mathrm{FCH})$ is performed [15]. It has an integrated procedure FL to choice $\mathrm{CH}$ based on energy, node awareness, and attentiveness at the time of computation. The $\mathrm{CH}$ selects the BS at each turn, determining the possibility that each node turns into a $\mathrm{CH}$, supported by fuzzy descriptors ("energy, concentration, and concentration"). The FL model has four stages: these stages are classified as fuzzification for the input descriptors is the first stage, rule evaluation is the second stage, aggregation of rules is the third stage, and the defuzzification is the final stage apart from that $\mathrm{FCH}$ is equated to the popular $\mathrm{LEACH}$ protocol; FND improved than Leach and increases network lifetime compared to LEACH.

\section{CHEF}

The paper [16] proposes a $\mathrm{CH}$ selection mechanism based on fuzzy logic (CHEF).Unlike LEACH, CHEF is deployed to reserve other nodes to $\mathrm{BS}$ and $\mathrm{CH}$ selections. At each turn, there could be notice the random values generated by the nodes like 0 to 1 and then compares the resulting value with a starting point. If the sample value is less than the probability value is considered using the fuzzy IF-THEN rule.

\section{LEACH-FL}

LEACH-FL is an advanced version of the LEACH by FL [17]. It has a comparable mechanism to the LCH, but the variables (parameters) set up to the battery charge level, the distance from the sink, and the nodes' density. The choice of $\mathrm{CH}$ is centered on the borehole, which must compute the probability that the nodes turn out to be $\mathrm{CH}$, just like $\mathrm{LCH}$. Network life with LEACH-FL is much enhanced than network life with LEACH.

\section{ICT2TSK}

"ICT2TSK is an advanced clustering algorithm that uses type 2 "Takagi-Sugeno-Kang (TSK) as the FL system" [18]. ICT2TSK is deployed to select $\mathrm{CH}$ and better manage regulatory ambiguities than the TSK FL Type 1 system. It assesses the network load by introducing a fixed radius of competition for each $\mathrm{CH}$, thus improving energy efficiency.

\section{SEP-FL}

"SEP-FL is an FL method that improves $\mathrm{CH}$ selectivity in a heterogeneous WSN" [19]. This is an improved version of SEP that depends on $\mathrm{CH}$ selectivity by balancing the residual energy probabilities of each node. SEP-FL offers a more extended stability period and lower volatility and intensifies the service life of the units. The SEP-FL network increases service life and significantly reduces power consumption compared to the other three algorithms.

\section{EAUCF}

The author by [20] proposes a fuzzy algorithm for energy-aware in an asymmetric or unequal cluster called EAUCF. The goal is to reduce $\mathrm{CH}$ energy consumption in clusters because they are close to BS or have less battery power.

\section{DFLC}

For the challenging of network lifetime and energy consumption in WSNs, the author has proposed distributed fuzzy logic-based clustering routing (DFLCR)[21] protocol. Related with similar protocols, the projected DFLCR protocol has reserved residual energy and local distance, significance into account. The DFLCR protocol is capable to choice the best node as $\mathrm{CH}$, and regulator the size of cluster. DFLCR protocol progresses the network lifetime. Model results illustration that the proposed DFLCR can increase network lifetime and progress the efficient of transmitting data.

$S I F$

In addition, fuzzy logic (FL) have been applied for solving various kind of problems in WSN .SIF is a "swarm intelligence protocol based on FL routing and RE" [22], distance to sink, and distance from the set center to select appropriate CHs. Using the SIF Fuzzy C-Means (FCM)

Clustering algorithm to group all sensor nodes into balanced clusters, $\mathrm{CH}$ is selected according to the Mamdani fuzzy inference system. SIF integrates a group of hybrid intelligence called FA-SA. The FA-SA Firefly Behavior (FA) algorithm and the robust local search algorithm are SA-based, and both are deployed to optimize the fuzzy system fuzzy rule base table.

\section{FBUC}

Asymmetric grouping based on FBUC or fuzzy is an enhanced version of the EAUCF [23]. FNB says FBUC has better energy depletion and better network life than the other two algorithms and on different stages.

\section{EEDCF}

The authors proposed: EEDCF [24] protocol called "energy-efficient distributed clustering based on fuzzy" [24] is an differential evolution algorithm in WSN the main intention 
of the algorithm to saving the energy while clustering based routing operation and Cluster head selection. Network lifetime EEDCF is improved than EADEEFG and DFLC. In both cases, it has better FND, HND, and LND values.

\subsubsection{Genetic Algorithms}

The GA ispotentially serves as a more efficient way to recalculate the routing topology of the WSN in real-time, compelling into account the distance among the nodes and the energy level of each node. In the GA models, improvement is made by carrying out fitness tests on new structures to select the best population. This optimization algorithm is benefited by a random search and routing data. GA has been flexible in dealing with dynamic problems and can be deployed successfully.

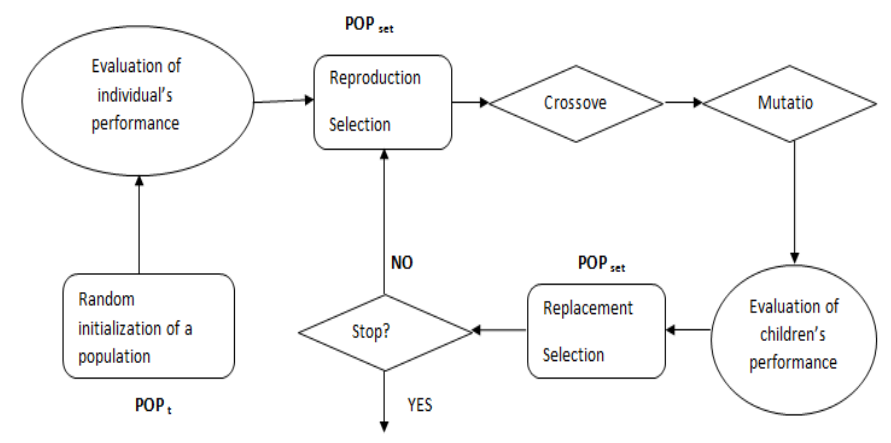

Figure 6: Structure of Genetic Algorithms

The GA global scheme comprises of 6 main elements: such as 1 . Initial set of configurations; 2 .Chromosome coding / decoding work; 3 .Gene operator (mutation, crossing,); 4.evaluation function (suitability); 5.selection algorithm (tournament, roulette,);

\section{Wazed et al}

The paper by [25] has Suggested "genetic algorithm to extend the lifespan of sensor networks at two levels." It plans to collect data from relay nodes and can considerably prolong the life of a relay node. The relay node behaves as a $\mathrm{CH}$ and receives data from the nodes fitting to its cluster. Every single relay node must transmit data to a common node or another relay or BS node. The Suggested routing algorithms find the right way to compare with the base station MHTDM and MTEM.

\section{Bayrakli, and al.}

The author by[26] has Suggested the GABEEC algorithm. The protocol is executed in two stages: first, Configuration, and next Steady State. It starts with nodes called $\mathrm{CHs}(\mathrm{CHs})$ that are unsystematically generated in the network. Using GA, the solution finds the applicable count of $\mathrm{CH}$ and their locations.

\section{Hamidouche et.al}

The author [27] has suggested genetic algorithm for multipath routing based on energy entropy WSN (GAEMW). The main idea of the protocol is to discover the minimum residual compel of each routing node in the multipath routing selection process. This will let down the remaining energy of the node.

\section{Abo-Zahhad et.al}

The work [28] imparted another adaptive energy efficiency clustering hierarchy protocol based on a genetic algorithm (GAEEP) to increase lifetime efficiency and improve WSN stability. The protocol aspires to extend the service life of WSNs by obtaining the optimal number of $\mathrm{CHs}$ and their locations based on reducing the energy consumption of sensor nodes using GA.

\section{Hussain et al.}

The work of [29] is intended to decrease energy depletion and intensify network life. The limits deployed here are "BS distance, data transfer, cluster distance and energy level [29]. The Suggested algorithm is equaled with LEACH, HCR-1, and HCR-2". The obtained outcomes have recorded that the network lifetime is better by refining the number of active nodes than the additional algorithms.

\section{Liu et.al}

The author proposes a GA based adaptive clustering algorithms to perform optimal thresholding for cluster formation in this regards LEACH-GA is an improved version of LEACH based on GA [30]. Furthermore to the LEACH configuration and static state, LEACH-GA method outstrips DT, LEACH and MTE, in case of network lifetime; meanwhile the exploit of the best probability concedes optimal energy-efficient clustering. The Suggested algorithm identifies the optimal threshold probability for clustering. The results show that by increasing the number of live nodes per cycle can improve network lifetime compared to leech-GA leeches.

\subsubsection{Neural Network}

An ANN is a complex system of parallel or distributed processing components called neurons or nerve cells that are incorporated into a graph topology. Neurons are connected using weighted connections called synapses. ANNs are computational algorithms that can master complex mapping concerning inputs and outputs using a supervised learning method to complete the classification of input data using an unsupervised method. 


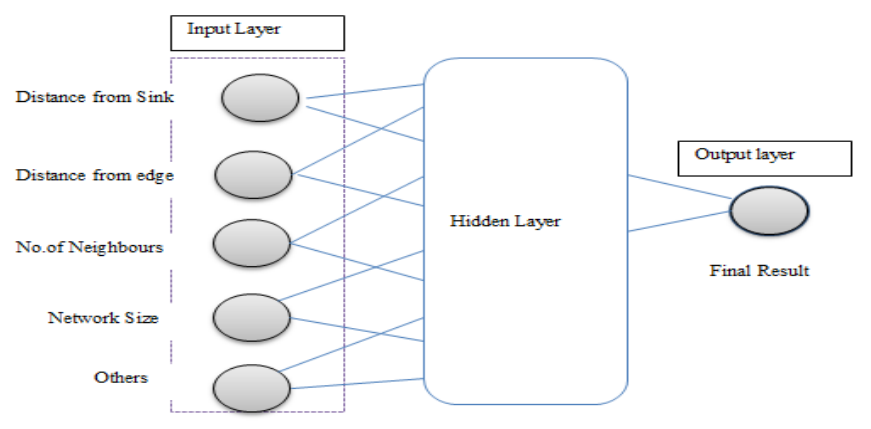

Figure 7. Basic neural Network Model in WSN

\section{Cordina\&Debono [31]}

In this paper, the author demonstrates "a clusterbased routing algorithm based on fuzzy ART neural networks" to proliferate these systems' lifetime. "The results show that the centralized version of LEACH, Leech, and the selforganizing map (SOM) improve energy savings by $79.6 \%$, $17.1 \%$, and $22.4 \%$ (for the first node matrices) compared to the neural network. - Based on the clustering algorithm accordingly". Besides, this article discusses the use of a sensitive filtering algorithm centered on a base station to reduce the amount of transmission data. This use results in a higher network lifetime.

\section{Nehra, $N$ et.al [32]}

In this paper, the author proposes clustering based on neural networks and energy-efficient WSN routing to increase network life. In the projected system, the challenging is modeled as "linear programming (LP)" with special constraints. $\mathrm{CH}$ selection is performed using adaptive learning in $\mathrm{NN}$, resulted in routing and data transmission.

\subsubsection{Reinforcement Learning}

In recent years, ML technology has attracted the attention of many involved in research and studies. RL is a sub-domain of ML. This attempt to use computer programs to create models or procedures from large data sets.

\section{WenjingGuo et.al}

The author [33] offers a routing protocol based on augmentation learning to optimize network life in all aspects defined by the author. The RL routing protocol uses the intelligent amplification learning algorithm's advantages to find the optimal routing path for data transmission. Factors such as link distance, remaining capacity, and the number of hops at the well are taken into account when determining the compensation function. The simulation results show that a routing protocol based on amplification learning optimizes network lifetime and improves energy efficiency in three respects compared to efficient energy routing, "BEER, Routing, and MRL-SCSO."
GuoWJ et.al

The author by [34] has proposed, "an intelligent routing algorithm called RLLO". It uses the predominance of reinforcement learning (RL) and takes into account the residual power and hopping calculation to define the reward function". This means an even distribution of energy consumption and improved packet delivery at no extra cost. This Suggested algorithm is compared to "Energy-Aware

Routing (EAR) and EAR Enhanced (I-EAR)." The result of the model can significantly increase network lifetime and packet delivery.

\section{CLIQUE}

CLICK [35], a data clustering method, saves the power of $\mathrm{CH}$ selection through machine learning. That way, nodes can independently determine based on $\mathrm{CH}$. We believe that the absence of a true $\mathrm{CH}$ distribution is free and consumes up to $25 \%$ less energy compared to invalid random $\mathrm{CH}$, combined with the acquisition of dynamic network functions such as battery backup.

\subsubsection{Swarm Intelligence}

Swarm intelligence can be useful for problems such as finding the minimum path. It is not always a straight line between endpoints; this could be the path along the edges of the chart. "SI systems are classified as optimization of the number of particles, ant colonies, and bee colonies."

\section{Particle Swarm Intelligence}

"Particle swarm (PSO) optimization" is a stochastic algorithm that is well known in the literature on swarm intelligence. This algorithm is inspired by the behavior of a bird flock in the wild. The interaction of birds in the flock is formed mathematically. In PSO, each solution has three characteristics: such as Position, Speed or velocity, and Fitness value.

\section{PSO-C}

The author [36] provides an energy-efficient grouping of WSNs using a "particle pool optimization (PSO)" algorithm implemented in a base station. The author defines a new cost function to reduce cluster distance and optimize network power consumption simultaneously. "PSO-C was compared to traditional LEACH clusters and its LEACH-C upgrade version in two different scenarios". PSO-C can also increase network lifetime by refining the number of active nodes in both cases.

\section{Kuila\& Jana}

The [37] author proposes "WSN power-efficient algorithms for clustering and routing," based on the example of the TSO. In an efficient particle coding method, the routing algorithm has also been developed with a compromise between transmission distance and multifunctional fitness 
function. At this time, the clustering algorithm takes the energy efficiency of the nodes into account because of the Load Balance Strategy. "The proposed algorithm is compared to the GA approach and two GLBCA and LDC clustering strategies." The proposed OSP solution would improve network life significantly.

\section{PSO-HC}

The author by [38] has proposed a new "centralized PSO protocol for hierarchical clustering (PSO-HC) WSN”. Our objective is to improve network life by reducing the number of active $\mathrm{CHs}$ and enhanced network scalability by means of two-way communication between sensor nodes and related CHs. The effect on the relation of WSN clusters of the use of a practical network and energy consumption model has been studied. Extensive simulations indicate that the PSO-HC

Overcomes average power and performance common cluster sensor network protocols.

\section{MPSICA}

The author of[39] proposes a "multicellular immune collaboration (MPSICA) algorithm that provides an intelligent routing rehabilitation scheme. MPSICA can handle KD design paths from the next super node and the optimal path from the super node to the sink from each source node." It can also explore optimal routing strategies and solve cloning, highfrequency mutations, clone selection operations that enhance error tolerance and the efficiency of intra-cluster data transmission. The "MPSICA-based protocol" will interact efficiently with the smart bio heuristic routing recovery system, thereby extending WSN life.

\section{TPSO-CR}

Two LP formulations for problem grouping and routing were proposed by the author by [40]. "TSO's clustering protocol provides a balance between energy consumption, network coverage and data reliability. Especially in comparison to the famous LEACH, EHE-LEACH, EEHC, LEACH-C, PSO-C und GA-C, the TSO-based process was proposed." The study found that TPSO-CR had less clustered nodes than any of the other protocols in both cases

\section{Ant Colony Optimization}

Routing can be carried out via ACO, using "neighbor discovery information, routing and data transmission and information on route management." To fix the problem of probabilistic optimization ACO replicates the action of a real ant colony - to find the best direction in a weighted diagram. Mapping of the shortest way to optimize energy-sensitive routing is used by artificial ants.

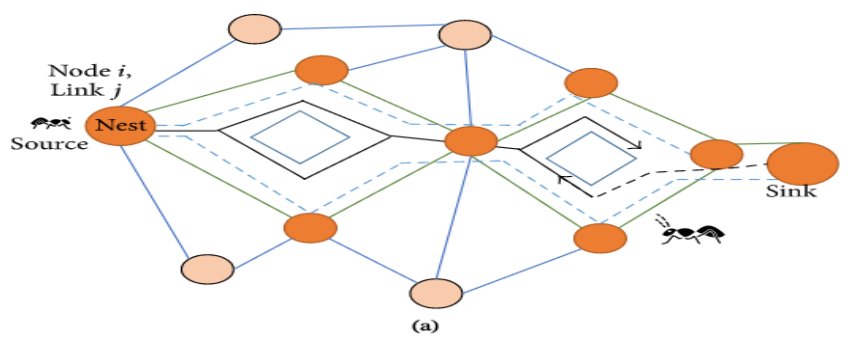

Figure 8: (a) Forward ant stochastically selects the path

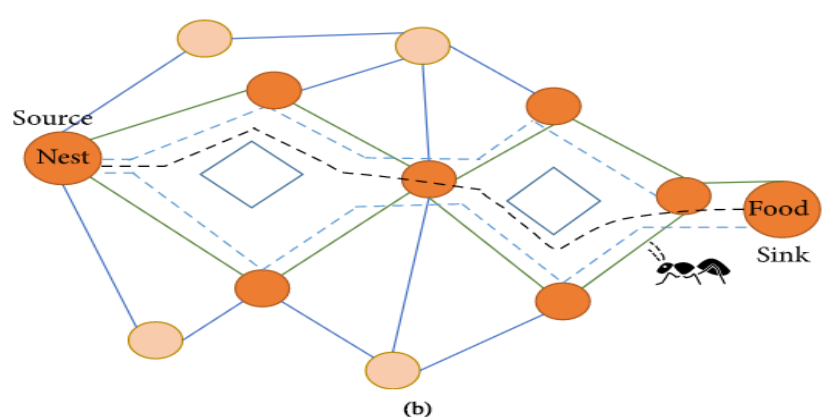

Figure 8: (b) Backward ant stochastically selects the path.

Figure 8 depicts a simplified version of the ACO in which the ants find the best path between the food source and the nest. Works in the WSN area use ACO-based clustering algorithms to improve sensor networks' performance to a very great extent.

\section{T-ANT}

This paper [41] has introduced a practical selforganization approach to effective disaster management. This approach attempts to use an "energy-efficient clustering approach". Using a clustering approach, self-organization led to inefficient network disaster management and energy efficiency strategies. Clustering is a method that helps diminish energy consumption.

\section{$E B A B$}

Author [42] "Energy Balanced Ant-Based Routing Protocol (EBAB). EBAB describes a new adaptive dynamic routing algorithm based on simple biological ants that can find and find ways to connect". The EBRD produces a disproportionate amount to balance the group's energy depletion. Clusters near the base station needless significant cluster sizes to increase network uptime. If the size of the cluster is not the same, EBAB operations, do not need geographical location, arrival direction, and topology regulator.

ACO-C

The author [43] demonstrates an adaptive routing protocol for optimizing WSN data collection to reduce network congestion and reduce data duplication to progress energy efficiency. "Ant colony optimization routing" has 
delivered an important mechanism to study the optimal parameters for data collection and channel selection. The traditional clustering order refers to dynamic low-power routing using adaptive protocol schemes. The "generalized ACO along with a new dynamic routing protocol is designed to optimize the capabilities of clusters and $\mathrm{CHs}$ to distribute power evenly across all sensor nodes to improve power source generation". Therefore, the critical energy competence of a WSN is improved by balancing efficient routing and energy consumption.

\section{ACA-LEACH}

The author [44] in this paper has suggested a "lowenergy adaptive clustering hierarchy analysis of the Wireless Sensor Network (WSN) cluster routing algorithm (LEACH) based on the ant colony algorithm (ACA)". The ACA also applied an "inter-cluster routing mechanism" and sought the best path from the $\mathrm{CHs}$ to the base station. The algorithm has inexpensive routing, self-organization, and multipath functionality.

\section{MRP}

The author [45] recommended a "new multipath routing protocol based on clustering and ACO." By presenting an objective role for dynamic grouping, MRP improves data collection efficiency, thus reducing energy consumption. The performance evaluation present MRP allows for improved load balancing and reduced energy consumption, followed by an increase in network life.

\section{Bee Colony Optimization}

The "artificial bee colony algorithm" provides energy savings and improved reliability effects for MWSN data collection by optimizing the mobile sink path, reducing energy consumption, improving efficiency and stability of network data collection, and extending network life.

\section{$P B C-C P$}

The author [46] suggested a "Proficient bee colony Clustering protocol (PBC-CP) based on an artificial bee colony algorithm". Using the "PBC-CP approach", we took the elements required for head selection, such as node power, node degree, and distance from the base station to the node. The transfer of data from the $\mathrm{CH}$ to the base station chooses an energy-efficient method that added reduces the sensor network's energy consumption.

\section{IABCOCT}

Author [47], the main goal of topology control, energy-saving, and extending the life cycle of sensor networks is essential to increase its service life. Met heuristic optimization algorithms are recognized as optimal to facilitate $\mathrm{CH}$ selection and clustering process. It is suggested to improve the clustering technique based on the optimization of artificial bee colonies (IABCOCT)", gaining the explosion benefits of a grenade and sofa operator to ensure the appropriate grouping process and selection of grouping heads. The result of the IABCOCT simulation reveals the reduction of the mortality rate of the sensor nodes is reduced to increase the service life of the network. The results are better than "EPSOCT, HCCHE and CCT" and are affected by different cycles of relative power, sink speed, average power consumption, and network routing overhead.

\section{$A B C-C$}

A new [48] new energy-efficient clustering approach centered on an $\mathrm{ABC}$ has been shown to extend network life. The ABC algorithm simulates the functionality of $\mathrm{ABC}$ ICWAQ. Due to this, the artificial bee colony algorithm is deployed successfully deployed in cluster techniques, which simulates the rational behavior of bee clusters. The Suggested protocol uses the efficient and fast search functionality of the "ABC ICWAQ algorithm to optimize the aggregation of nodes in the $\mathrm{CH}$ selection process that identifies the routing gateways." The accomplishment of the ABC algorithm clustering was compared to protocols centered on "LEACH and TSO." The protocol does not extend the duration of the ICWAQ network.

\section{Bee-Sensor-C}

"Bee Sensor-C" is an energy-efficient and scalable multi-channel routing protocol based on dynamic cluster and bee group behavior [49]. It adopts an improved multipath architecture strategy, achieving balanced energy depletion in the network, reducing overhead routing and refining network scalability and performance, as well as its lifetime. The suggested algorithm is associated to the "Bee Sensor inspired routing protocol Bee sensor [50], IEEABR [51], and Flood Forward Ant Routing FF-Ant [52]". The results revealed that the Bee-Sensor-C consumes less power, but has a higher packet transfer rate than the other three protocols.

\section{BeeSwarm}

Manns et al. [53] suggested a "CI-based met heuristic for energy-efficient hierarchical routing" called bee Sworm. It consists of three stages: "Beacluster, BeeSearch, and Beaccarrier. In the configuration phase (Becluster), a similar ABC approach is deployed to create optimal clusters called Bee Zones (BZ) and to select the appropriate $\mathrm{CH}$ called Zonal Head (ZH)". The Suggested beam worm approach is evaluated and compared with MRP routing protocols and with the variable performance parameter ERP algorithm [54]. The results show that Beaver outperforms other routing protocols in terms of total packet delivery and packet delivery speed. We observe that the bee worm uses less energy than the other two protocols, thus increasing the network's life. 


\section{Evaluation of the Optimized Clustering Approaches}

This section compares several clustering algorithms optimized in terms of machine learning for some essential parameters; data delivery speed, continuous power consumption in the network lifetime account, and algorithm scalability. Type of centralized or distributed method, integration or non-integration of data collection techniques, and uniformity of nodes.

Data delivery rate concerns to the volume of data received by a $\mathrm{BS}$ or a receiving node, rendering to the number of data sent by other nodes. This indicator is significant as it helps to get summary of data loss during transmission and reliability throughout the communication.

The energy consumption parameter is one of the essential metrics to assess clustering algorithms. The depletion of node energy affects the sensor network's lifetime because if the node involves more communication or transactions, it may reduce energy and decrease the network's lifetime.

Subsequently, our investigation concentrates on large-scale WSN, which deliberates the scalability of the existing algorithms, which classifies the number of nodes in the network for the investigation, it recommends more number of nodes i.e., more than 500 nodes make attention to the further investigation.
Clustering algorithms are implemented in two approaches, i.e., Centralized and extensive. Where in the Centralized approach, BS plays a vital role in making decisions. In this regard when ML adapts to the network, BS should broadcast the essential information i,e RE and Location information to its plotting nodes and computes, whereas in the distributed system all

Network with nodes showing the same characteristics are called Homogeneity. For cluster $\mathrm{CH}$ selection in such a network with nodes possessing unique characteristics is selected as $\mathrm{CH}$

The radio model in the Sensor network estimates the amount of energy consumed by the node in the sensor network field. In this connection, the Clustering approach integrates this radio model into the network and calculates the node energy levels while transmitting for receiving and sending.

To consider the fault tolerance of the presented algorithms, we have defined multipath metrics. Multipath implementation algorithms have a higher error tolerance than the others unless the path to the BS is no longer available; a side road can access BS. This parameter allows the algorithm to avoid a complete network failure CA deployed by a node failure.

\subsection{Performance comparison of clustering algorithms}

Table 1(a).Optimized clustering approaches comparisons

\begin{tabular}{|c|c|c|c|c|c|}
\hline $\begin{array}{c}\text { Optimized clustering } \\
\text { approaches. }\end{array}$ & $\begin{array}{c}\text { ML/CI } \\
\text { paradigms }\end{array}$ & $\begin{array}{c}\text { Data Delivery } \\
\text { rate }\end{array}$ & $\begin{array}{c}\text { Data } \\
\text { Aggregation }\end{array}$ & $\begin{array}{c}\text { Energy } \\
\text { Consumption }\end{array}$ & Scalability \\
\hline $\mathrm{FCH}[15]$ & FL & Average & No & High & Low \\
\hline CHEF[16] & FL & Average & YES & High & Medium \\
\hline LEACH-FL[17] & FL & NA & NA & High & Low \\
\hline ICT2TSK[18] & FL & NA & NA & low & Medium \\
\hline SEP-FL[19] & FL & NA & NA & Average & low \\
\hline EAUCF[20] & FL & NA & YES & Low & Low \\
\hline DFLC[21] & FL & Low & NA & Average & Medium \\
\hline SIF[22] & FL/SI & Average & No & Low & Medium \\
\hline FBUC[23] & FL & NA & NA & Low & Low \\
\hline EEDCF[24] & FL & Average & No & Average & Low \\
\hline GA[25] & GA & Average & No & Average & Medium \\
\hline GABEEC[26] & GA & Average & Yes & Low & Low \\
\hline GAEMW[27] & GA & Average & No & Average & Medium \\
\hline GAEEP[28] & GA & Average & & Average & Low \\
\hline GA[29] & GA & Average & No & Average & Medium \\
\hline GA[30] & GA & Average & Yes & Average & Medium \\
\hline $\mathrm{NN}$ [31] & $\mathrm{NN}$ & Average & Yes & Average & Low \\
\hline
\end{tabular}




\begin{tabular}{|l|c|c|c|c|c|}
\hline NN [32] & NN & Average & & Average & Low \\
\hline RL & RL & Average & Yes & Low & Medium \\
\hline RLLO[34] & RL & Average & No & Average & Low \\
\hline CLIQUE[35] & RL & Average & Yes & Low & Medium \\
\hline PSO-C[36] & RL & Average & Yes & Average & Low \\
\hline SI[37] & RL & Low & No & Average & Low \\
\hline PSO-HC[38] & PSO & Average & Yes & Average & High \\
\hline MPSICA[39] & PSO & Average & Yes & high & High \\
\hline TPSO-CR[40] & PSO & High & Yes & Average & High \\
\hline T-ANT[41] & ACO & High & Yes & Average & Low \\
\hline EBAB[42] & ACO & Low & & Average & High \\
\hline ACO-C[43] & ACO & High & Yes & Average & Low \\
\hline ACA-LEACH [44] & ACO & Average & No & High & Medium \\
\hline PBC-CP[46] & ABC & Low & Yes & Low & High \\
\hline MRP[45] & BCO & Average & Yes & Average & Average \\
\hline ABC-C[48] & SI & High & Yes & High & High \\
\hline BEE-S-C[49] & SI & high & Yes & Low & High \\
\hline BeeSensor[50] & SI & High & Yes & Low & High \\
\hline IEEABR[51] & SI & High & Yes & Low & High \\
\hline $\begin{array}{l}\text { Flood Forward Ant Routing } \\
\text { FF-Ant [52] }\end{array}$ & ACO & High & Yes & Low & High \\
\hline BeeSwarm [53] & SI & High & Yes & Low & Medium \\
\hline ERP[54] & High & Yes & Low & Medium \\
\hline
\end{tabular}

Table 1(b) Optimized clustering approaches comparisons

\begin{tabular}{|l|c|c|c|c|}
\hline \multicolumn{1}{|c|}{ Optimized clustering approaches. } & Nature & Network type & Multi-hop & multipath \\
\hline FCH[15] & Centralized & Homogeneous & No & No \\
\hline CHEF[16] & distributed & Homogeneous & No & No \\
\hline LEACH-FL[17] & Centralized & Homogeneous & No & No \\
\hline ICT2TSK[18] & Centralized & Homogeneous & No & yes \\
\hline SEP-FL[19] & Centralized & Homogeneous & Yes & No \\
\hline EAUCF[20] & Distributed & Homogeneous & Yes & Yes \\
\hline DFLC[21] & Distributed & Homogeneous & Yes & Yes \\
\hline SIF[22] & Distributed & Homogeneous & Yes & Yes \\
\hline FBUC[23] & Distributed & Homogeneous & Yes & Yes \\
\hline EEDCF[24] & Distributed & Homogeneous & Yes & Yes \\
\hline GA[25] & Centralized & Homogeneous & Yes & NA \\
\hline GABEEC[26] & Distributed & Homogeneous & No & No \\
\hline GAEMW[27] & Centralized & Homogeneous & Yes & NA \\
\hline GAEEP[28] & Distributed & Homogeneous & Yes & Yes \\
\hline GA[29] & Centralized & Heterogeneous & Yes & NA \\
\hline GA[30] & Distributed & Homogeneous & No & NA \\
\hline NN [31] & Distributed & Homogeneous & No & Yes \\
\hline NN [32] & Distributed & Homogeneous & Yes & Yes \\
\hline RL & Distributed & Homogeneous & Yes & Yes \\
\hline RLLO[34] & Distributed & Homogeneous & No & Yes \\
\hline
\end{tabular}




\begin{tabular}{|l|c|c|c|c|}
\hline CLIQUE[35] & Distributed & Homogeneous & Yes & Yes \\
\hline PSO-C[36] & Centralized & Homogeneous & No & No \\
\hline SI[37] & Distributed & Homogeneous & No & No \\
\hline PSO-HC[38] & Centralized & Homogeneous & Yes & No \\
\hline MPSICA[39] & Distributed & Heterogeneous & Yes & Yes \\
\hline TPSO-CR[40] & Centralized & Homogeneous & Yes & Yes \\
\hline T-ANT[41] & Distributed & Homogeneous & Yes & Yes \\
\hline EBAB[42] & Distributed & Homogeneous & Yes & Yes \\
\hline ACO-C[43] & Centralized & Homogeneous & No & Yes \\
\hline ACA-LEACH [44] & Distributed & Homogeneous & Yes & Yes \\
\hline PBC-CP[46] & Distributed & Homogeneous & Yes & Yes \\
\hline MRP[45] & Distributed & Homogeneous & Yes & Yes \\
\hline ABC-C[48] & Distributed & Homogeneous & Yes & Yes \\
\hline BEE-S-C[49] & Distributed & Homogeneous & Yes & Yes \\
\hline BeeSensor[50] & Distributed & Homogeneous & Yes & Yes \\
\hline IEEABR[51] & Distributed & Homogeneous & Yes & Yes \\
\hline Flood Forward Ant Routing FF-Ant [52] & Distributed & Heterogeneous & Yes & Yes \\
\hline BeeSwarm [53] & Distributed & Heterogeneous & Yes & Yes \\
\hline ERP[54] & Distributed & Heterogeneous & Yes & Yes \\
\hline
\end{tabular}

\section{Mathematical Approaches for WSN Lifetime Enhancement}

The authors by [55] have introduced a mathematical model that effectively validates the unconventional path between the receiver and the transmitter for data transmission. The unconventional path shall include the relay node in the standby method and shall conform to the mathematical asymmetry specified in lemma 1 . The choice of any other node that serves as a relay result in data collection using network coding, which leads to excessive energy replacement. The power dissipation from the receiver is not insignificant compared to the transmitter dissipation. The node transmits data in standby mode only without data processing.

Table 2.Simulation Parameter Settings Model for WSN

\begin{tabular}{|l|l|}
\hline Parameters & Values \\
\hline The initial energy of a sensor node $E_{0}$ & $5 \mathrm{~J}$ \\
\hline Duty Cycle $\overline{T_{r}}$ & $10 \%$ \\
\hline Duration of a data period $T_{r}$ & $10 \mathrm{~s}$ \\
\hline The energy consumption rate for idle listening & $0.88 \mathrm{~mJ}$ \\
\hline Data transmission rate B & $512 \mathrm{~Kb} / \mathrm{s}$ \\
\hline Size of a data packet $\tau$ & $400 \mathrm{bits}$ \\
\hline
\end{tabular}

In the first level radio model, the radio transmits energy and sends and receives a packet in succession. When a node relay serves as a power source, the cost is relatively low compared to sending the similar packet from the same node because there is no data collection in the node. Thus, here the mathematical equation for transmitting the length of packets over the entire length " $\mathrm{k}$ " $\mathrm{d}$ " is considered only significant and given.

$$
E_{T x}(k, d)=e_{\text {amp }} * d^{\lambda} * k+E_{\text {elec }} * k
$$

With $E_{T x}->E_{R x}$

Equation (1) is scattered to control the transmitter and has a value of $50 \mathrm{~nJ} /$ bit, equal to the power scatter and $100 \mathrm{pJ} /$ bit / $\mathrm{m} 2$ by the transmission amplifier.

Lemma1. Conversely data transmission between two sensor nodes disjointed by a "two-dimensional Euclidian distance d", dispersing energy is reduced by immediately putting the data transfer into standby mode with "twodimensional Euclidean distances" from two source nodes as $d_{1} d_{2 "}$ and" and nourishing the relation $d_{1}^{2}+d_{2}^{2}<d^{2}$ .Proof.

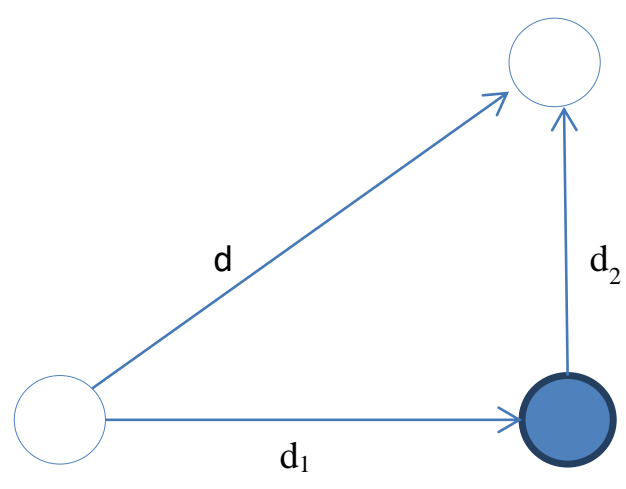

Figure 9. Routing among the nodes and relay node

$$
E_{T x}(k, d)=e_{\text {amp }} * x^{2} * k+E_{\text {elec }} * k
$$


Transmission energy dissipation for direct routing between the sources nodesis $E_{T x}(k, d)=\mathrm{e}_{\mathrm{d}} * \mathrm{~d}^{2} * \mathrm{k}$

Sleep and relay node between two nodes is calculated as

$$
E_{T 2 x}(k, d)=e_{d} * d_{1}^{2} * k+e_{d} * d_{2}^{2} * k_{(4)}
$$

For low power consumption through the another path, the disparity that must be satisfied is

$$
E_{T 2 x}(k, d)_{<} E_{T 1 x}(k, d)_{(5)}
$$

By substituting the corresponding values of the energy transmitted by Equations 3 and 4, the energy consumption is significantly compact by treating the node in standby mode as a relay node, if the condition specified in Equation 5 is fulfilled. However, given the estimated calculations, an error can be added, and it should be noted that this mathematical model is fully proven when the estimate is fulfilled.

$$
d_{1}^{2}+d_{2}^{2}<d
$$

\section{Machine Learning Adaptability For WSN's}

Machine learning can naturally resolve different challenges in WSNs and increase their performance considerably.

Few application of ML in WSNs to prolong the network lifetime are:

- Identifies the optimal number of nodes reach the target area coverage

- To predict the volume of energy to be picked within a particular time slot to improve the WSN lifetime.

- Accurate localization with ML

- $\quad$ isolate the faulty nodes with ML

- Data dimensionality reduction at the sensor or $\mathrm{CH}$ level using ML.

\subsection{Supervised Learning}

The supervised learning method is deployed toresolve several the issues for WSNs, such as localization [55], Coverage Problems [84], Data aggregations [85], Event detection [86], routing and target tracking [87].

\section{Support Vector Machines:}

SVM is an alternate option for solving uncontrolled or curved optimization problems. In the case of WSN, when the congestion occurs in the network near the nodes at the sink. The "support vector machine (SVM)" is a useful classification algorithm for machine learning. Using SVM, its applicability to the localization problems and addresses the border and coverage-hole problems effectively [56].

\section{Decision Trees}

A "decision tree" is a learning algorithm that practices tree-like graphs to ideal and evaluates discrete functions. $\mathrm{CH}$ selection is major process to enhance the network life time in WSNs with the help of decision tree to elect the best node [57]. Furthermore the practice of decision trees provides localization techniques and overall strategy to classify critical characteristics of link reliability, comprising loss rate, average recovery time, and average failure time,

\section{Neural Networks}

$\mathrm{NN}$ are one of the most widespread learning algorithms and are built using decision units to learn data. Cascade circuits are often referred to as perceptual and radial basis functions. Decision unit cascade chains help identify linear and complex data associations. However, the learning practice, which involves several cascade circuits, is very intensive in computing [58].

\section{$K$-nearest neighbor ( $k$-NN)}

"K-NN is a supervised learning algorithm," the sample test data is categorized according to the labels of the nearby data models. Missing or unknown test samples are calculated by calculating nearby averages. This approach [58] is essential to detect the fault and anomalies deployed the $\mathrm{k}$ NN algorithm.

\section{Bayesian Learners}

Bayesian language learners [59] accurately estimate the movement (speed) of sensor nodes without prior knowledge of mobility information in the WSN sensor node. Using these mobility estimation methods, develop routingbased mobility prediction methods and analytical mobility prediction models.

\subsection{Unsupervised learning}

Unsupervised learning gain knowledge from experience, there is no particular training model build. Furthermore, there are no labels provided. These algorithms are deployed in WSN for clustering and data aggregation [60]. "K-means. Clusteringand component analysis" plays are essential in the approach

\section{Principal Component Analysis:}

Data aggregation plays a major role in WSN as far as it reduces power consumption and increases the scalability of the network. The "Principal Component Analysis (PCA)" [61]is suitable technique to preprocessing data i.e dimensionality reduction from sensor networks. PCA-guided routing algorithm exploring the principal component analysis (PCA) approach, which reduces energy depletion and extends network lifetime by understanding the objective of decreasing the amount of distances between the nodes and the cluster centers in a WSN network. 


\section{K-Means Clustering}

"K-means clustering algorithmic [62]" is one of the most popular models of all partition-based algorithms for clustering data. In order to construct the clusters, K-means algorithm balances and reduces energy consumption by selecting the CH's with high energy to prolong lifetime.

\section{Reinforcement Learning}

"Reinforcement Learning (RL)" is deployed in a wide range of WSN projects, including collaborative communications, routing, and flow control, so sensors and sensing nodes can observe optimal operations and execution network in the appropriate operating environment. And improvements in application performance.

This is called trial and error model, i.e., learning from the environment, this model is flexible and adapts to environment changes, quickly relearn, manages productively with flexible backgrounds. This model is simple to apply and develop. Insignificant to adequate processing and memory needs, and it can be applied thoroughly. RL for WSN majorly concentrates on different issues such as Clustering protocols, Medium Access protocols, and routing protocols; further Hyper parameter optimization is also addressed within the scope of reinforcement learning, specifically for architectural network design and application-level cooperation among nodes. Q-learning [63] is a useful algorithm for amplifying learning algorithms that are well known for WSN routing problems.

Table 3.ML based Solutions in Wireless sensor network

\begin{tabular}{|c|c|c|c|c|c|c|c|}
\hline ML Techniques & Routing & C\&D & LQE & NL & $\mathbf{L E}$ & EM & QoS \\
\hline Naïve Bayes & & & & & [64] & & \\
\hline SVM & & & & [65] & [64] & & \\
\hline Matric-map & [66] & [67] & [66][67] & & & & \\
\hline Multilayer Perceptron & & & & & {$[68][64]$} & [68] & \\
\hline Neural Network & [69] & [70] & & & & [70] & [70] \\
\hline $\begin{array}{l}\text { Semi-supervised } \\
\text { Learning }\end{array}$ & & [71] & & $\begin{array}{c}{[72][71][6} \\
5]\end{array}$ & & & \\
\hline Hybrid learning & & [64] & & & & & [73][64] \\
\hline $\begin{array}{l}\text { Unsupervised } \\
\text { Learning }\end{array}$ & [74] & & & & [74] & [74] & {$[74]$} \\
\hline $\begin{array}{l}\text { Reinforcement } \\
\text { Learning }\end{array}$ & $\begin{array}{l}{[74][75][76]} \\
{[77][78][79]} \\
\end{array}$ & [79] & & & [77][78][79] & $\begin{array}{c}{[77][78][79]} \\
{[80]} \\
\end{array}$ & [76][79][77] \\
\hline Game Theory & {$[81][82]$} & [82][83] & & & [80] [81] [83] & {$[82][83]$} & [81] \\
\hline $\begin{array}{l}\text { Computational } \\
\text { Intelligence }\end{array}$ & [81] [84] & {$[81]$} & & & & {$[82]$} & \\
\hline
\end{tabular}

- C\&D: Clustering \&Data Aggregation

- LQE: Link Quality Estimation

- NL: Node Localization

- LE: Lifetime Extension

- EM: Energy Management

\section{Hierarchical clustering}

This clustering techniques related objects that require a fixed "top-down or bottom-up order". The hierarchical clustering deployed to resolve various difficulties in WSNs, majorly it address the issues like data aggregation [88], synchronization [89], mobile sink [90], [91], and energy harvesting [92]".

\section{Fuzzy-c-means clustering}

It's also called as soft clustering, there are few techniques were deployed to formulate the clusters depends on the similarity measurements, i.e "intensity, distance, or connectivity". FCM technique deployed to solve several potential issues in WSNs i.e "localization [93], [94], connectivity [95], and mobile sink [96]".

Table 4. Possibility to add a few parameters for cluster performance improvement

\begin{tabular}{|l|c|c|c|}
\hline Specification type & $\begin{array}{c}\text { K-means } \\
\text { clustering }\end{array}$ & $\begin{array}{c}\text { Fuzzy c- } \\
\text { means }\end{array}$ & Hierarchical \\
\hline Clustering speed & Fast & Slow & Fast \\
\hline Accuracy & Low & High & High \\
\hline $\begin{array}{l}\text { Accuracy of } \\
\text { prediction }\end{array}$ & High & Low & Low \\
\hline Quality & High & Moderate & Moderate \\
\hline Sensitivity & High & Low & Low \\
\hline $\begin{array}{l}\text { Randomness in } \\
\text { results }\end{array}$ & Moderate & Moderate & Good \\
\hline Performance & High & Moderate & Moderate \\
\hline
\end{tabular}




\section{Future Directions and Challenges}

Several interesting aspects may be explored further by Machine Learning-based Optimized Hierarchical Routing Protocols for WSN Lifetime: present days ML based applications are drastically improved to address the WSN issues. Furthermore there are contradictory researchers have different expectations, application scenarios, and priorities for the use of ML algorithms. These differences are a major challenge for researchers to work together so that common in the community for the WSN machine.

Some of the research challenges to prolong the network lifetime using optimized cluster-based routing protocols using machine learning in WSN are

- Optimal selection of secure and energy $\mathrm{CH}$ selection in WSN

- Reduce Energy Consumption to extend the stability and network lifetime [97]

- To achieve better coverage and connectivity performance in WSN.

- The appropriate procedure for initialization and the number of iterations to achieving the reasonable computational cost.

- Link quality prediction using machine learning.

- Resource management using machine learningi.e.Developing"lightweight and distributed message passing techniques, online learning algorithms, and hierarchical clustering patterns."

\section{Conclusion}

In recent years, ML techniques have seen significant advances in WSN. The review make available for detailed analysis and possibility on the adaptability of ML techniques which support the optimized Hierarchical Routing Protocols in WSN for Network lifetime improvement. To attain this, we explore the several parameters that are taken into consideration for optimized $\mathrm{CH}$ selection and formation. Furthermore, perhaps a more meaningful comparison among the existing optimized clustering methods and ML-based approaches has been present. Furthermore also presents the several routing approaches towards" scalability, energy consumption, and the amount of data delivered to the BS are enlightened. Eventually, our review enlightens the scholars, readers, and authors to gain the knowledge to work with

ML-based Optimized Hierarchical Routing Protocols to prolong the WSN lifetime.

As future works, we plan to optimal cluster formation and classify an optimal selection of secure and energy $\mathrm{CH}$ selection in WSN through relay node communication. The study for future work, would be discuss the "spectrumefficient clustering studies in practical large wireless sensor networks to address the energy issues in WSN."

\section{Acknowledgment}

I thank Dr.Kavitha for their expertise and assistance throughout all aspects of our study and for their help in writing the review manuscript.

\section{References}

[1] Shafiq, Maryam, et al "Systematic Literature Review on Energy Efficient Routing Schemes in WSN Asurvey." Mobile Networks and Applications (2020): 14.

[2] Awan, Khalid Mahmood, et al. "Energy efficient cluster based routing algorithm for wireless sensors networks." 2018 International Conference on Advancements in Computational Sciences (ICACS). IEEE, 2018.

[3] Rehmani, Mubashir Husain, and Al-Sakib Khan Pathan. "Emerging communication technologies based on wireless sensor networks: current research and future applications."(2016).

[4] Pradeepa, K., W. Regis Anne, and S. Duraisamy. "Design and implementation issues of clustering in wireless sensor networks." International Journal of Computer Applications 47.11 (2012).

[5] Wategaonkar, Dhanashri N., and Vivek S. Deshpande. "Characterization of reliability in WSN." 2012 World Congress on Information and Communication Technologies. IEEE, 2012.

[6] Yong-Min, Liu, Wu Shu-Ci, and Nian Xiao-Hong. "The architecture and characteristics of wireless sensor network." 2009 International Conference on Computer Technology and Development. Vol. 1. IEEE, 2009.

[7] Low, Arthur. "Evolution of wireless sensor networks for industrial control." Technology Innovation Management Review 3.5 (2013).

[8] Liu, Xuxun. "A survey on clustering routing protocols in wireless sensor networks." sensors 12.8 (2012): 1111311153.

[9] Jain, Neeraj, P. Sinha, and S. K. Gupta. "Clustering protocols in wireless sensor networks: A survey." International Journal of Applied Information System (IJAIS) 5.2 (2013).

[10] Bhanderi, M., and H. Shah. "Machine Learning for Wireless Sensor Network: A Review, Challenges and pplications." Adv.Electron. Electr. Eng 4 (2014): 475486.

[11] Junhai, Luo, Xue Liu, and YeDanxia. "Research on multicast routing protocols for mobile ad-hoc networks." Computer Networks 52.5 (2008): 988-997.

[12] Sirsikar, Sumedha, and KalyaniWankhede. "Comparison of clustering algorithms to design new clustering approach." Procedia Computer Science 49 (2015): 147154.

[13] Solaiman,

Basma,andAlaaSheta."Computationalintelligencefor wireless sensor networks: Applications and clustering algorithms." International Journal of ComputerApplications 73.15 (2013): 1-8. 
[14] .Liu, Xuxun. "A survey on clustering routing protocols in wireless sensor networks." sensors 12.8 (2012): 11113-11153.

[15] Gupta, Indranil, Denis Riordan, and SrinivasSampalli. "Cluster-head election using fuzzy logic for wireless sensor networks." 3rd Annual communication networks and services research conference (CNSR'05). IEEE, 2005.

[16] Kim, Jong-Myoung, et al. "CHEF: cluster head election mechanism using fuzzy logic in wireless sensor networks." 2008 10th International Conference on Advanced Communication Technology. Vol. 1. IEEE, 2008.

[17] Ran, Ge, Huazhong Zhang, and Shulan Gong. "Improving on LEACH protocol of wireless sensor networks using fuzzy logic." Journal of Information \&Computational Science 7.3 (2010): 767-775.

[18] Zhang, Feng, Qi-Ye Zhang, and Ze-Ming Sun. "ICT2TSK: An improved clustering algorithm for WSN using a type-2 Takagi-Sugeno-Kang Fuzzy Logic System." 2013 IEEE Symposium on Wireless Technology \& Applications (ISWTA). IEEE, 2013.

[19] Mostafa, Baghouri, ChakkorSaad, and HajraouiAbderrahmane. "Fuzzy logic approach to improving Stable Election Protocol for clustered heterogeneous wireless sensor networks." J. Theor. Appl. Inf. Technol 14 (2014): 112-116.

[20] Bagci, Hakan, and AdnanYazici. "An energy aware fuzzy approach to unequal clustering in wireless sensor networks." Applied Soft Computing 13.4 (2013): 17411749 .

[21] Alaybeyoglu, Aysegul. "A distributed fuzzy logicbased root selection algorithm for wireless sensor networks." Computers \& Electrical Engineering 41 (2015): 216-225.

[22] Zahedi, ZeynabMolay, et al. "Swarm intelligence based fuzzy routing protocol for clustered wireless sensor networks." Expert Systems with Applications 55 (2016): 313-

[23] Logambigai, Rajasekar, and ArputharajKannan. "Fuzzy logic based unequal clustering for wireless sensor networks." Wireless Networks 22.3 (2016): 945-957.

[24] Zhang, Ying, et al. "Fuzzy-logic based distributed energy-efficient clustering algorithm for wireless sensor networks." Sensors 17.7 (2017): 1554.

[25] Wazed, Shamsul, et al. "Genetic algorithm based approach for extending the lifetime of two-tiered sensor networks." 2007 2nd International Symposium on Wireless Pervasive Computing. IEEE, 2007.

[26] Bayrakl1, Selim, and SenolZaferErdogan. "Genetic algorithm based energy efficient clusters (gabeec) in wireless sensor networks." Procedia Computer Science 10 (2012): 247-254.

[27] Hamidouche, Ranida, ZiboudaAliouat, and AbdelhakMouradGueroui. "Genetic algorithm for improving the lifetime and QoS of wireless sensor networks." Wireless Personal Communications 101.4 (2018): 2313-2348.

[28] Abo-Zahhad, Mohammed, et al. "A new energyefficient adaptive clustering protocol based on genetic algorithm for improving the lifetime and the stable period of wireless sensor networks." International Journal of Energy, Information and Communications 5.3 (2014): 4772.

[29] Hussain, Sajid, Abdul WaseyMatin, and Obidul Islam. "Genetic algorithm for hierarchical wireless sensor networks." J. Networks 2.5 (2007): 87-97.

[30] Liu, Jenn-Long, and Chinya V. Ravishankar. "LEACHGA: Genetic algorithm-based energy-efficient adaptive clustering protocol for wireless sensor networks." International Journal of Machine Learning and Computing 1.1 (2011): 79.

[31] Cordina, Mario, and Carl James Debono. "Maximizing the lifetime of wireless sensor networks through intelligent clustering and data reduction techniques." 2009 IEEE Wireless

[32] Communications and Networking Conference. IEEE 2009.

[33] Nehra, Neeraj Kumar, ManojKumar, and R. B. Patel. "Neural network based energy efficient clustering and routing in wireless sensor networks." 2009 First International Conference on Networks \& Communications. IEEE, 2009.

[34] Guo, Wenjing, Cairong Yan, and Ting Lu. "Optimizing the lifetime of wireless sensor networks via reinforcement-learning-based routing." International Journal of Distributed Sensor Networks 15.2 (2019): 1550147719833541.

[35] Guo WJ, Yan CR, Gan YL, et al. "An intelligentrouting algorithm in wireless sensor networks based on reinforcement learning", ApplMechMater ; 678: 487493,2014

[36] Forster, Anna, and Amy L. Murphy. "CLIQUE: Rolefree clustering with Q-learning for wireless sensor networks." 2009 29th IEEE International Conference on Distributed Computing Systems. IEEE, 2009.

[37] Latiff, NM Abdul, Charalampos C. Tsimenidis, and Bayan S. Sharif. "Energy-aware clustering for wireless sensor networks using particle swarm optimization." 2007 IEEE 18th international symposium on personal, indoor and mobile radio communications. IEEE, 2007.

[38] Kuila, Pratyay, and Prasanta K. Jana. "Energy efficient clustering and routing algorithms for wireless sensor networks: Particle swarm optimization approach." Engineering Applications of Artificial Intelligence 33 (2014): 127-140.

[39] Elhabyan, Riham S., and Mustapha CE Yagoub. "PSO HC: Particle swarm optimization protocol for hierarchical clustering in Wireless Sensor Networks." 10th IEEE international conference on collaborative computing: networking, applications and works haring. IEEE, 2014.

[40] Ding, Yongsheng, et al. "MPSICA: An intelligent routing recovery scheme for heterogeneous wireless sensor networks." Information Sciences 308 (2015): 49-60.

[41] Elhabyan, Riham SY, and Mustapha CE Yagoub. "Two tier particle swarm optimization protocol for clustering and routing in wireless sensor network." Journal of Network and Computer Applications 52 (2015): 116128. 
[42] Selvakennedy, S., S. Sinnappan, and Yi Shang. "TANT: a nature-inspired data gathering protocol for wireless sensor networks." Journal of Communications 1.2 (2006): 22-29.

[43] Wang, Lin, Ruihua Zhang, and ShichaoGeng. "An energy-balanced ant-based routing protocol for wireless sensor networks." 2009 5th International Conference on Wireless Communications, Networking and Mobile Computing. IEEE, 2009.

[44] Ziyadi, Morteza, KeyvanYasami, and BahmanAbolhassani. "Adaptive clustering for energy efficient wireless sensor networks based on ant colony optimization." 2009 Seventh

[45] Annual Communication Networks and Services Research Conference. IEEE, 2009.

[46] Wang, Guifeng, Yong Wang, and Xiaoling Tao. "An ant colony clustering routing algorithm for wireless sensor networks." 2009 Third International Conference on Genetic and Evolutionary Computing. IEEE, 2009.

[47] Yang, Jing, et al. "A multipath routing protocol based on clustering and ant colony optimization for wireless sensor networks." Sensors 10.5 (2010): 4521-4540.

[48] Pathak, Aruna. "A Proficient Bee Colony-Clustering Protocol to Prolong Lifetime of Wireless Sensor Networks." Journal of Computer Networks and Communications 2020 (2020).

[49] .Famila, S., and A. Jawahar. "Improved Artificial Bee Colony Optimization-Based Clustering Technique for WSNs." Wireless Personal Communications 110.4 (2020): 2195-2212.

[50] Karaboga, Dervis, SelcukOkdem, and CelalOzturk. "Cluster based wireless sensor network routing using artificial bee colony algorithm." Wireless Networks 18.7 (2012): 847-860.

[51] Cai, Xuelian, et al. "Bee-sensor-C: an energy-efficient and scalable multipath routing protocol for wireless sensor networks." International Journal of Distributed Sensor Networks 11.3 (2015): 976127.

[52] Saleem, Muhammad, and Muddassar Farooq. "Beesensor: a bee-inspired power aware routing protocol for wireless sensor networks." Workshops on Applications of Evolutionary Computation. Springer, Berlin, Heidelberg, 2007.

[53] Camilo, Tiago, et al. "An energy-efficient ant-based routing algorithm for wireless sensor networks." International workshop on ant colony optimization and swarm intelligence. Springer, Berlin, Heidelberg, 2006.

[54] Zhang, Ying, Lukas D. Kuhn, and Markus PJ Fromherz. "Improvements on ant routing for sensor networks." International Workshop on Ant Colony Optimization and Swarm Intelligence. Springer, Berlin, Heidelberg, 2004.

[55] Mann, Palvinder Singh, Satvir Singh, and Anil Kumar. "Computational Intelligence based metaheuristic for energy efficient routing in wireless sensor networks." 2016 IEEE congress on evolutionary computation (CEC). IEEE, 2016.

[56] Bara'a, A. Attea, and Enan A. Khalil. "A new evolutionary based routing protocol for clustered heterogeneous wireless sensor networks." Applied Soft Computing 12.7 (2012): 1950-1957.

[57] Banihashemian, Seyed Saber, FazlollahAdibnia, and Mehdi Sarram. "A new range-free and storage-efficient localization algorithm using neural networks in wireless sensor networks. "Wireless Personal Communications 98.1 (2018): 1547-1568.

[58] Kulkarni, Sanjeev R., Gábor Lugosi, and Santosh S. Venkatesh. "Learning pattern classification survey." IEEE Transactions on Information Theory 44.6 (1998): 2178-2206.

[59] Lu, Ching-Hu, and Li-Chen Fu. "Robust location-aware activity recognition using wireless sensor network in an attentive home." IEEE Transactions on Automation Science and Engineering 6.4 (2009): 598-609.

[60] Branch, Joel W., et al. "In-network outlier detection in wireless sensor networks." Knowledge and information systems 34.1 (2013): 23-54.

[61] Somaa, Fatma, et al. "A Bayesian model for mobility prediction in wireless sensor networks." 2016 International Conference on Performance Evaluation and Modeling in Wired and Wireless Networks (PEMWN). IEEE, 2016.

[62] Wang, Yong, Margaret Martonosi, and Li-ShiuanPeh. "Predicting link quality using supervised learning in wireless sensor networks." ACM SIGMOBILE Mobile Computing and Communications Review 11.3 (2007): 71-83.

[63] Rooshenas, Amirmohammad, et al. "Reducing the data transmission in wireless sensor networks using the principal component analysis." 2010 Sixth International Conference on Intelligent Sensors, Sensor Networks and Information Processing. IEEE, 2010.

[64] Jain, Bindiya, GursewakBrar, and Jyoteesh Malhotra. "EKMT-k-means clustering algorithmic solution for low energy consumption for wireless sensor networks based on minimum mean distance from base station." Networking communication and data knowledge engineering. Springer, Singapore, 2018. 113-123.

[65] Abolfazli, Zahra, and Mehdi Mahdavi. "A homogeneous wireless sensor network routing algorithm: An energy aware cluster based approach." 2014 22nd Iranian Conference on Electrical Engineering (ICEE). IEEE, 2014.

[66] Barnawi, Abdulaziz Y., and Ismail M. Keshta. "Energy management in wireless sensor networks based on naïve bayes, MLP, and SVM classifications: A comparative study." Journal of Sensors 2016 (2016).

[67] Yoo, Jaehyun, and H. Jin Kim. "Semisupervised location awareness in wireless sensor networks using laplacian support vector regression." International Journal of Distributed Sensor Networks 10.4 (2014): 265801.

[68] Wang, Yong, Margaret Martonosi, and Li-ShiuanPeh. "A supervised learning approach for routing optimizations in wireless sensor networks." Proceedings of the 2nd international workshop on Multi-hop ad hoc networks: from theory to reality. 2006 . 
[69] Wang, Yong, Margaret Martonosi, and Li-ShiuanPeh. "Predicting link quality using supervised learning in wireless sensor networks." ACM SIGMOBILE Mobile Computing and Communications Review 11.3 (2007): 71-83.

[70] Barnawi, Abdulaziz Y., and Ismail M. Keshta. "Energy management of wireless sensor networks based on multi-layer perceptrons." European Wireless 2014; 20th European Wireless Conference. VDE, 2014.

[71] Turčaník, M. "Neural Network Approach to Routing in Sensor Network." Advances in Military Technology, ISSN 2308 (1802): 71-82.

[72] Enami, Neda, et al. "Neural network based energy efficiency in wireless sensor networks: A survey." International Journal of Computer Science \&Engineering Survey 1.1 (2010): 39-53.

[73] Tripathi, Rajiv Kumar. "Base station positioning, nodes' localization and clustering algorithms for wireless sensor networks." Submit or recommend next manuscript to SCIRP and we will provide best service for you: Accepting pre-submission inquiries through Email, Facebook, LinkedIn, Twitter, etc. A wide selection of journals (inclusive of 9 subjects, more than 200 journals) Providing (2012).

[74] Yang, Bin, et al. "Localization algorithm in wireless sensor networks based on semi-supervised manifold learning and its application." Cluster Computing 13.4 (2010): 435-446.

[75] Sun, Yanjing, and Li Li. "Hybrid learning algorithm for effective coverage in wireless sensor networks." 2008 Fourth International Conference on Natural Computation. Vol. 5. IEEE, 2008.

[76] Zhang, Ying, and Qingfeng Huang. "A learning-based adaptive routing tree for wireless sensor networks." Journal of Communications 1.2 (2006): 1221.

[77] Forster, Anna, and Amy L. Murphy. "FROMS: Feedback routing for optimizing multiple sinks in WSN with reinforcement learning." 2007 3rd International Conference on Intelligent Sensors, Sensor Networks and Information. IEEE, 2007.

[78] Förster, Anna, et al. "An efficient implementation of reinforcement learning based routing on real WSN hardware." 2008 IEEE International Conference on Wireless and Mobile Computing, Networking and Communications. IEEE, 2008.

[79] Guo, Wen Jing, et al. "An intelligent routing algorithm in wireless sensor networks based on reinforcement learning." Applied Mechanics and Materials. Vol. 678. Trans Tech Publications Ltd, 2014.

[80] Oddi, Guido, Antonio Pietrabissa, and Francesco Liberati"Energy balancing in multi-hop Wireless Sensor Networks: an approach based on reinforcement learning." 2014 NASA/ESA Conference on Adaptive Hardware and Systems (AHS). IEEE, 2014.

[81] Kiani, Farzad, et al. "Efficient intelligent energy routing protocol in wireless sensor networks." International Journal of Distributed Sensor Networks 11.3 (2015): 618072.

[82] AlTabbakh, Shahinaz M., et al. "Energy management of wireless sensor network based on modeling by game theory approaches." Proceedings of the IEEE European Modelling Symposium (EMS'15). 2015.

[83] Attiah, Afraa, et al. "An evolutionary game for efficient routing in wireless sensor networks." 2016 IEEE Global Communications Conference (GLOBECOM). IEEE,2016.

[84] Gemeda, KetemaAdere, Gabriele Gianini, and MulugetaLibsie. "An evolutionary cluster-game approach for Wireless Sensor Networks in noncollaborative settings." Pervasive and Mobile Computing 42 (2017): 209-225.

[85] Yang, Liu, et al. "A game theoretic approach fobalancing energy consumption in clustered wireless sensor networks." Sensors 17.11 (2017): 2654.

[86] Sun, Wei, et al. "End-to-end data delivery reliability model for estimating and optimizing the link quality of industrial WSNs." IEEE Transactions on Automation Science and Engineering 15.3 (2017): 1127-1137.

[87] Song, Xin, et al. "DLRDG: distributed linear regression based hierarchical data gathering framework in wireless sensor network." Neural Computing and Applications 23.7-8 (2013): 1999-2013.

[88] Illiano, Vittorio P., and Emil C. Lupu. "Detecting malicious data injections in event detection wireless sensor networks." IEEE Transactions on Network and service management 12.3 (2015): 496-510.

[89] Braca, Paolo, et al. "Bayesian tracking in underwater wireless sensor networks with port-starboard ambiguity." IEEE Transactions on Signal Processing 62.7 (2014): 1864-1878.

[90] $\mathrm{Xu}, \mathrm{Xi}$, et al. "Hierarchical data aggregation using compressive sensing (HDACS) in WSNs." ACM Transactions on Sensor Networks (TOSN) 11.3 (2015): 125.

[91] Neamatollahi, Peyman, et al. "Hierarchical clustering task scheduling policy in cluster-based wireless sensor networks." IEEE Transactions on Industrial Informatics 14.5 (2017): 1876-1886.

[92] Zhang, Ruonan, et al. "NDCMC: A hybrid data collection approach for large-scale WSNs using mobile element and hierarchical clustering." IEEE internet of thin journal 3.4 (2015): 533-543.

[93] Zhang, Ruonan, et al. "A hybrid approach using mobile element and hierarchical clustering for data collection in WSNs." 2015 IEEE Wireless Communications and Networking Conference (WCNC). IEEE, 2015.

[94] Reddy, M. Jagadeeswara, P. Suman Prakash, and P. Chenna Reddy. "Homogeneous and heterogeneous energy schemes for hierarchical cluster based routing protocols in WSN: a survey." Proceedings of the third international conference on trends in information, telecommunication and computing. Springer, New York, NY, 2013.

[95] Zhu, Fang, and Junfang Wei. "Localization algorithm for large-scale wireless sensor networks based on FCMTSR-support vector machine." International Journal of Distributed Sensor Networks 12.10 (2016): 1550147716674010.

[96] Qin, Jiahu, et al. "Distributed \$ k \$-means algorithm and fuzzy \$ c \$-means algorithm for sensor networks 
based on multiagent consensus theory." IEEE transactions on cybernetics 47.3 (2016): 772-783.

[97] Nayak, Padmalaya, and AnuragDevulapalli. "A fuzzy logic-based clustering algorithm for WSN to extend the network lifetime." IEEE sensors journal 16.1 (2015): 137-144.

[98] Wang, Jin, et al. "Particle swarm optimization based clustering algorithm with mobile sink for WSNs." Future Generation Computer Systems 76 (2017): 452-457.

[99] Omar, Dina, and Ahmed M. Khedr. "SEPCS: Prolonging Stability Period of Wireless Sensor
Networks using Compressive Sensing." International Journal of Communication Networks and Information Security 11.1(2019): 1-6. 